\title{
Vascular Response to Modified Widman Flap Surgery in Monkeys*
}

\author{
Raul G. Caffesse, $†$ Walter A. Castelli, $\ddagger$ and Carlos E. Nasjleti.§
}

\begin{abstract}
REVASCULARIZATION OF THE periodontal tissues was studied after flap surgery. Modified Widman flap procedures were performed in four young Rhesus monkeys. All premolars and molars were involved. Experimental periods covered from 1 day to 90 days. The animals were sacrificed by exsanguination and perfused through the carotid arteries with a combined solution of equal parts of India ink and 10\% buffered formalin solution. After fixation and decalcification, part of the blocks were processed to obtain cleared specimens following the Spalteholtz method. The remaining blocks were processed for routine histologic examination. The findings, mainly from the cleared specimens, showed that 1 day after surgery the supraperiosteal vessels in the flap demonstrated an overt hyperemic response. Patency of the vessels in the periodontal membrane and cancellous bone was evident; however, vessels were not depicted in the exposed cortical bone, nor in the clot. Vascular continuity apical to the surgical detachment was observed. In 3 to 4 days vascular proliferation within the organizing clot was observed and anastomosis between the gingiva and cancellous bone or periodontal membrane started to develop. At 7 days anastomoses were evident and vasodilation was present throughout the tissues. At 15 days and thereafter, vascular organization started to decrease, and the only hyperemic foci remaining were associated with areas of delayed bone remodeling, cementum remodeling or persistent marginal inflammation.
\end{abstract}

Numerous reports have described the healing of periodontal flaps. Studies concerned with the healing of reverse bevel flaps and modified Widman flaps have been published from our laboratory. ${ }^{1,2}$ Further, the vascular response after flap surgery has been reported by different researchers. According to Kon et al. ${ }^{3}$ when a full thickness flap was raised in dogs, the vessels within the flap were not significantly altered, but increased vasodilation occurred. However, vascular disruption has been reported to be much more severe when a split thickness flap was performed. ${ }^{4}$

The present report will show the vascular changes observed after modified Widman flaps were performed in monkeys.

\section{MATERIALS AND METHODS}

Four young adult Rhesus monkeys (Macaca mulatta) with an average weight of 12 pounds were used. One week before the surgical procedure, they received a

* Supported in part by the Medical Research Division of the Veterans Administration.

† Professor and Chairman of Periodontics, The University of Michigan School of Dentistry, Ann Arbor, MI 48109.

$\ddagger$ Professor of Anatomy, The University of Michigan Medical School, Ann Arbor, MI 48109.

$\S$ Coordinator, Dental Research Program, Veterans Administration Medical Center, Ann Arbor, MI 48105. dental prophylaxis under general anesthesia with pentobarbital sodium (Nembutal), $30 \mathrm{mg} / \mathrm{kg}$ of body weight. In each monkey, modified Widman flap surgeries were performed on the four quadrants of the mouth. Each experimental condition was performed in duplicate as follows: monkeys 1 and 2 were used to evaluate healing after 1, 3, 4 and 7 days and monkeys 3 and 4 were used to evaluate healing after $15,21,30$ and 90 days after surgery. As previously described, ${ }^{5}$ good flap adaptation was achieved by means of interproximal independent sutures. Immediately after surgery, the animals were fed a soft diet, and had water ad libitum.

After 1 week, the sutures were removed and the teeth were polished. The animals were sacrificed by exsanguination under general anesthesia covering the experimental periods already mentioned. To visualize the vascularization of the surgical area better, the external carotid arteries were cannulated and perfused with a combined solution of equal parts of India ink (Pelikan carbon black) and $10 \%$ buffered formalin solution. After decapitation, blocks including the operated teeth and their supporting tissues, were fixed in $10 \%$ buffered formalin, and later decalcified in $4 \%$ formic acid. The blocks were cut buccolingually in four pieces. Two alternate pieces were cleared following the Spalteholtz method, ${ }^{6}$ while the other two pieces were prepared for routine histologic evaluation after embedding in paraffin, sectioning, and staining with hematoxylin and eosin. 

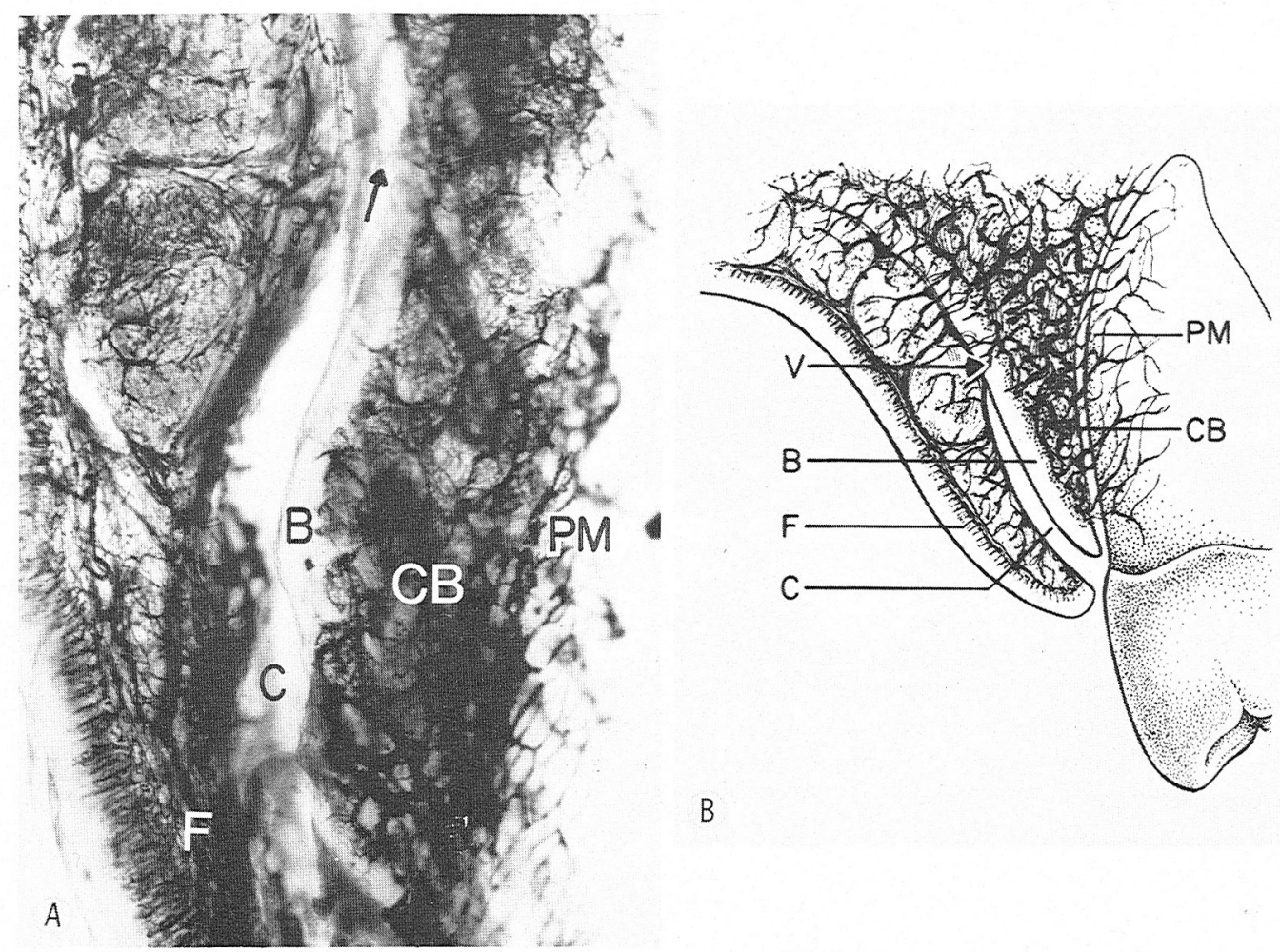

B

Figure 1. One day specimen. A. Cleared section. B. Schematic drawing of section. The flap (F) is separated from the underlying cortical bone (B) by the space left by the blood clot (C). Vessels on the cancellous bone (CB) and periodontal membrane (PM) are fully perfused. Blood vessels (arrow) are interconnecting the mucoperiosteal and cortical bone circulations at the bottom of the surgical attachment.

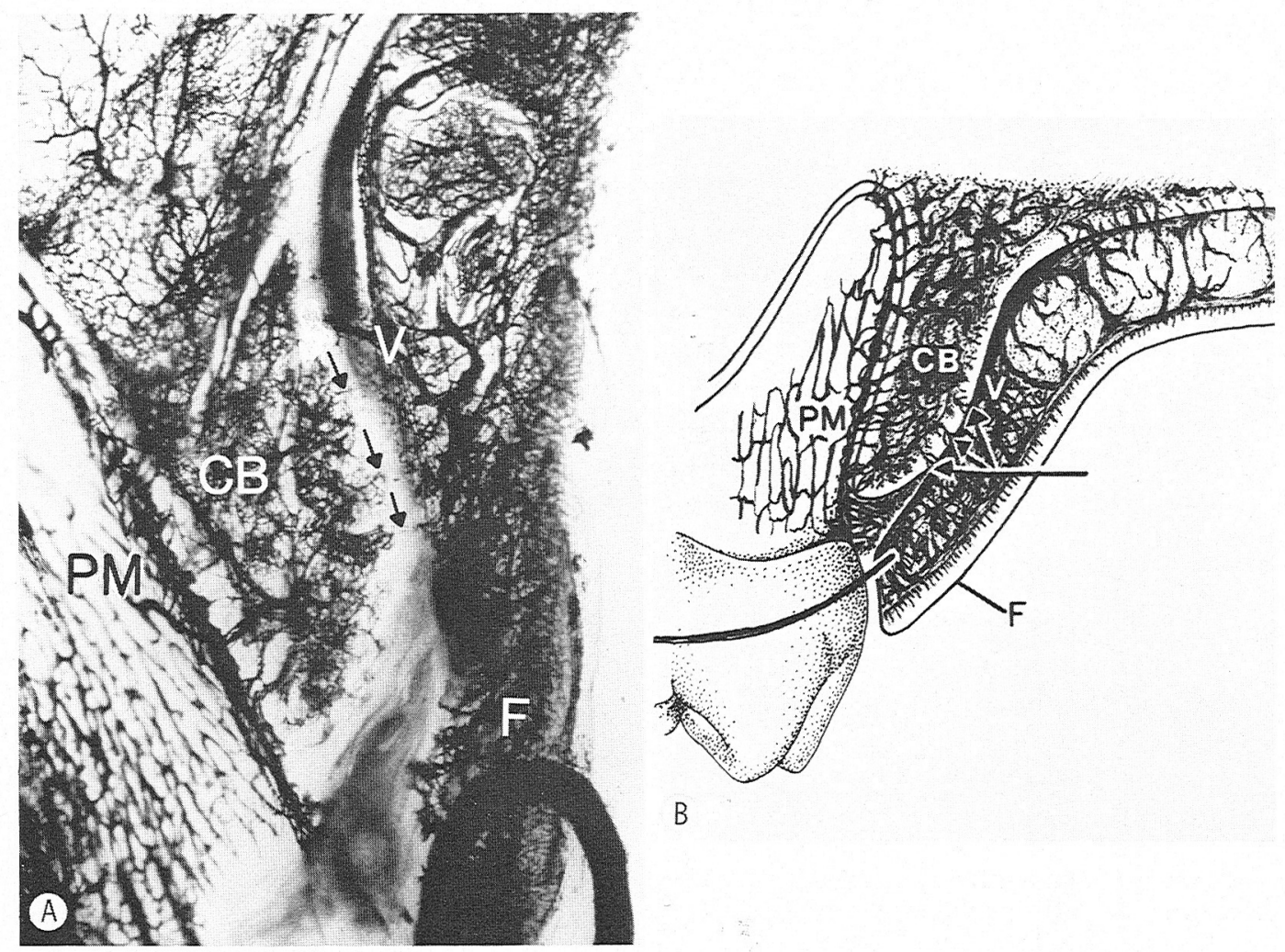

Figure 2. Three day specimen. A. Cleared specimen. B. Schematic drawing of section. Vascular proliferation (V) is seen associated with areas of good flap adaptation. Alveolar cortical vessels (arrow) are feeding the deep side of the intervening blood clot. Flap (F), cancellous bone (CB), periodontal membrane (PM). 


\section{RESULTS}

Although, the remaining histologic material was also analyzed and evaluated for comparison, the present report will be mainly devoted to findings from the cleared specimens.

One Day After Surgery. The separation of the mucoperiosteal flaps from the tooth and underlying structures is readily evident from the space left by the fibrinous blood clot that was present at this level. Vessels of the alveolar cortical bone are not fully patent in the cervical area. However, vessels of the adjacent cancellous bone and periodontal membrane are fully perfused. Vascular continuity is observed at the bottom of the surgical attachment where vessels connect the mucoperiosteal and cortical bone circulations. The vascular network within the mucoperiosteal flaps depicts a hyperemic response (Figs. 1A, 1B).

Three Days After Surgery. There is a vascular proliferation within the organizing blood clot at the bottom of the mucoperiosteal flaps, especially in areas where good flap adaptation was achieved. The vasculature of both vestibular and lingual flaps is hyperemic, and so are those of the adjacent cancellous bone and the cervical periodontal membrane. The deep side of the intervening blood clot is receiving blood through a few patent alveolar cortical vessels (Figs. 2A, 2B).

Four Days After Surgery. Good flap adaptation is evident in the specimens studied. Vascular proliferation in the organizing blood clot is advanced with anastomotic channels connecting the cancellous bone circulation with those of the flap and alveolar mucosa (Figs. 3A, 3B).

Seven Days After Surgery. The vascular network of the palatal flaps appears to be more profuse and extensive than those in the vestibular flaps. The vessels continue uninterrupted to the marginal gingiva where they join the highly hyperemic gingival vasculature. These gingival vessels are already showing continuity with those of the periodontal membrane.

Multiple alveolar cortical vessels are feeding the bed of both surgical flaps, originating from the cancellous bone. At the vestibular side, however, in areas where thin buccal bone is present separating the hyperemic periodontal vasculature and the mucoperiosteal flaps, the bone is already perforated by patent vascular channels connecting the periodontal vessels and those of the flap throughout (Figs. 4A, 4B, 4C).

Fifteen to Thirty Days After Surgery. After 15 days the periosteal vascular network adjacent to the surgical interface appears nearly normal. However, at the alveolar crest, there are localized hyperemic areas which are related to ongoing processes of bone remodeling (Figs. $5 \mathrm{~A}, 5 \mathrm{~B}, 5 \mathrm{C}$ ).

The 21 and 30 day specimens show a normal vascular network. Nevertheless, there are persistent areas of increased vascularity related to areas of alveolar crest remodeling, which are similar to those found in the 15 day specimens, or to marginal inflammatory foci, or to areas of cementum remodeling (Fig. 6).

Ninety Days After Surgery. The vasculature of the
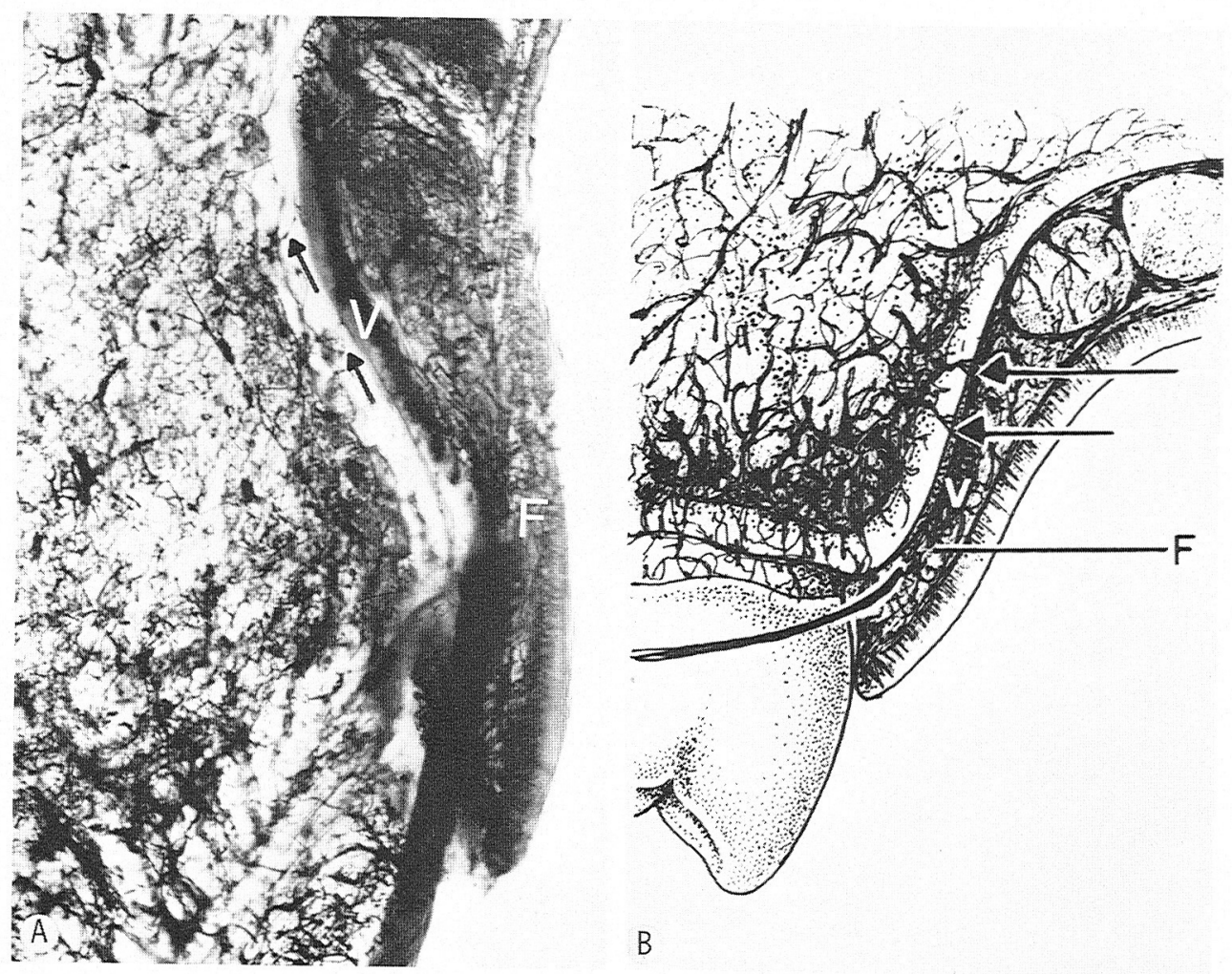

Figure 3. Four day specimen. A. Cleared section. B. Schematic drawing of the section. Vascular proliferation (V) is seen in the organizing blood clot. Anastomotic channels (arrows) are connecting the cancellous bone circulation with that of the flap (F). 

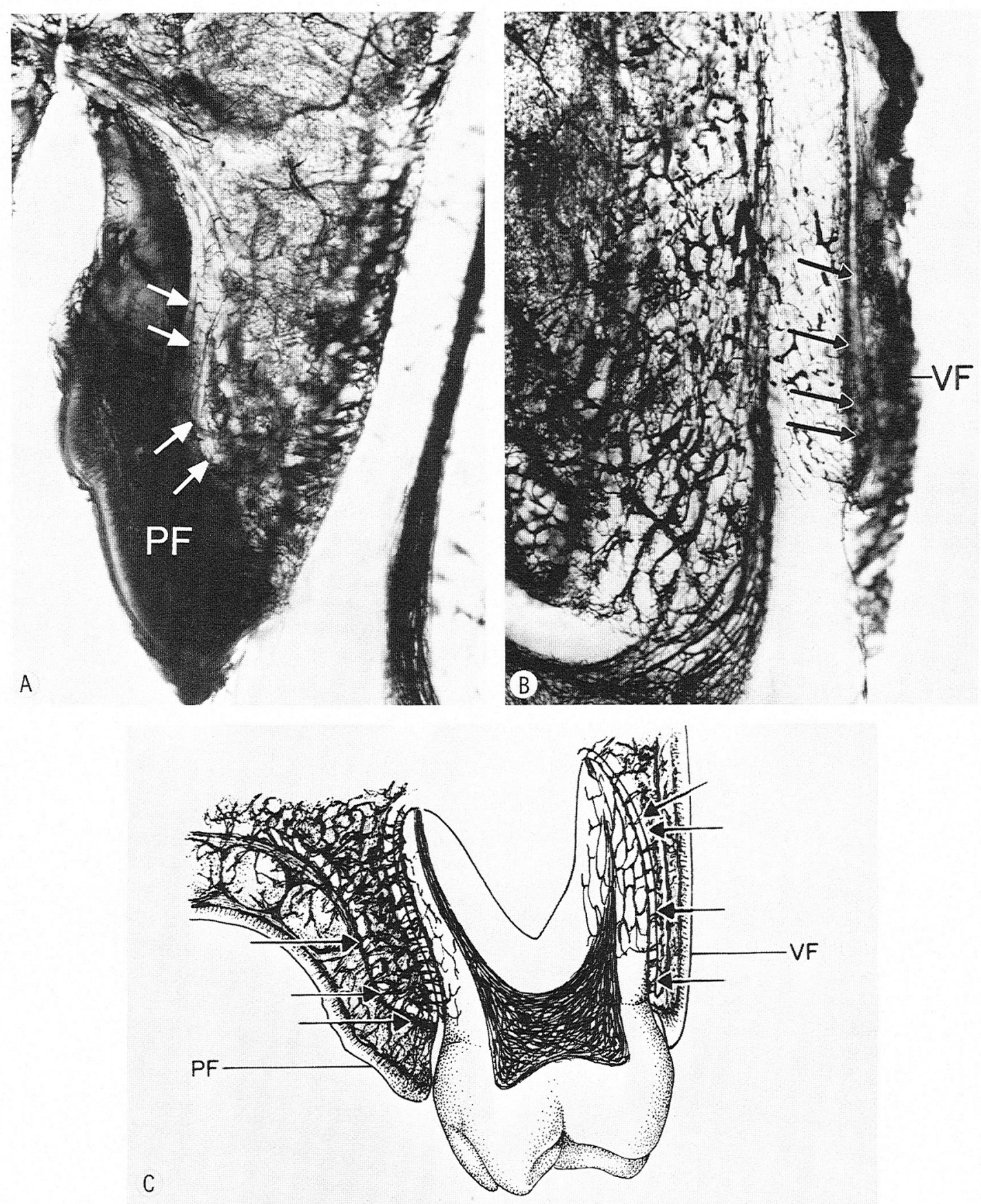

Figure 4. Seven day specimen. A and B. Cleared sections. C. Schematic drawing of the sections. Multiple alveolar cortical vessels (arrows) are feeding the bed of both surgical flaps. Short vessels are connecting the periodontal membrane and mucoperiosteal circulations on the vestibular side. Vestibular flap (VF), palatal flap (PF). 

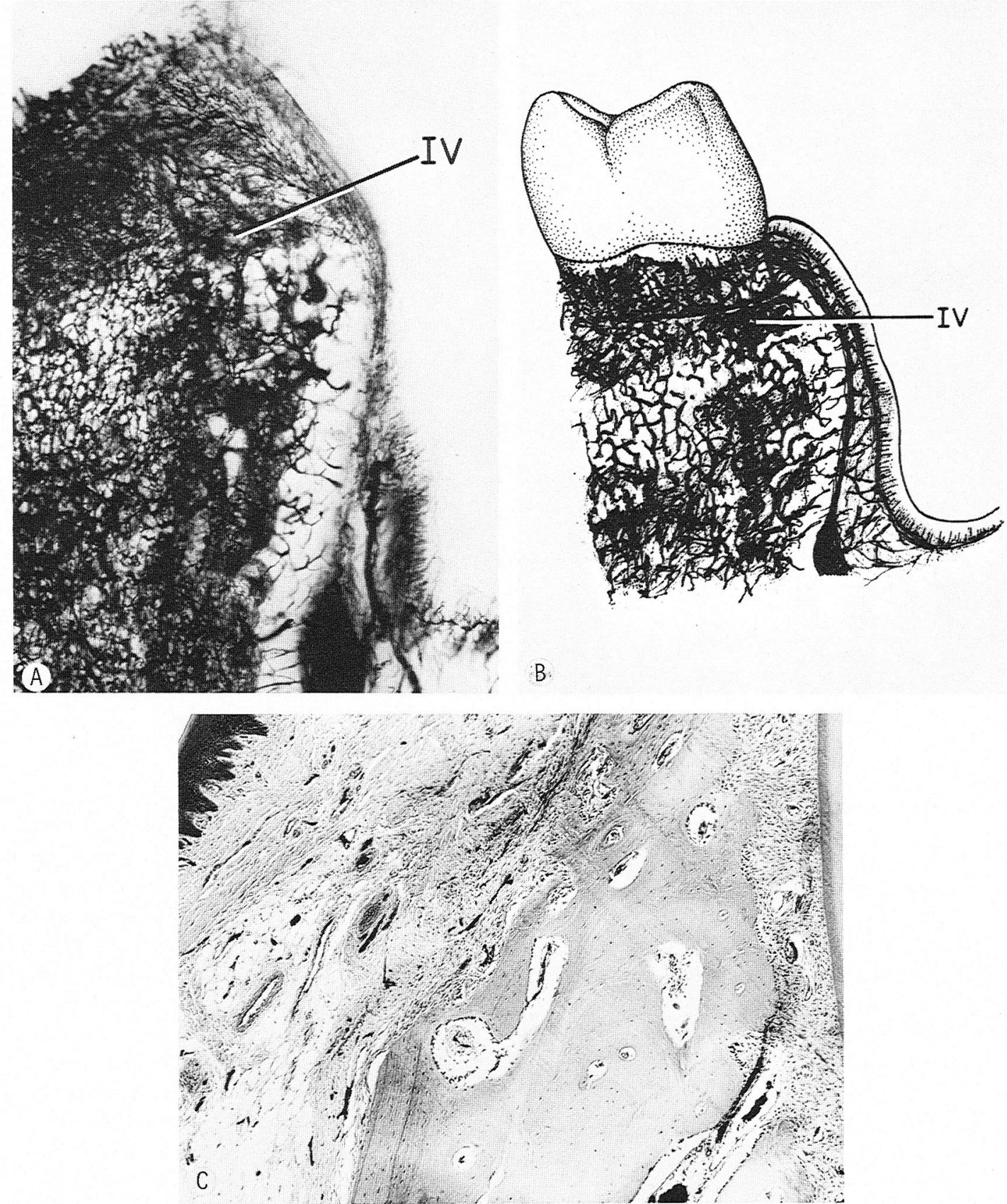

Figure 5. Twenty-one day specimen. A. Cleared specimen. B. Schematic drawing of the section. Vascular proliferation has been completed. Isolated areas of increased vascularity (IV) are associated with localized processes of bone remodeling. C. Histology with an area of ongoing bone remodeling, similar to that seen in the cleared specimen. Hematoxylin and eosin. 


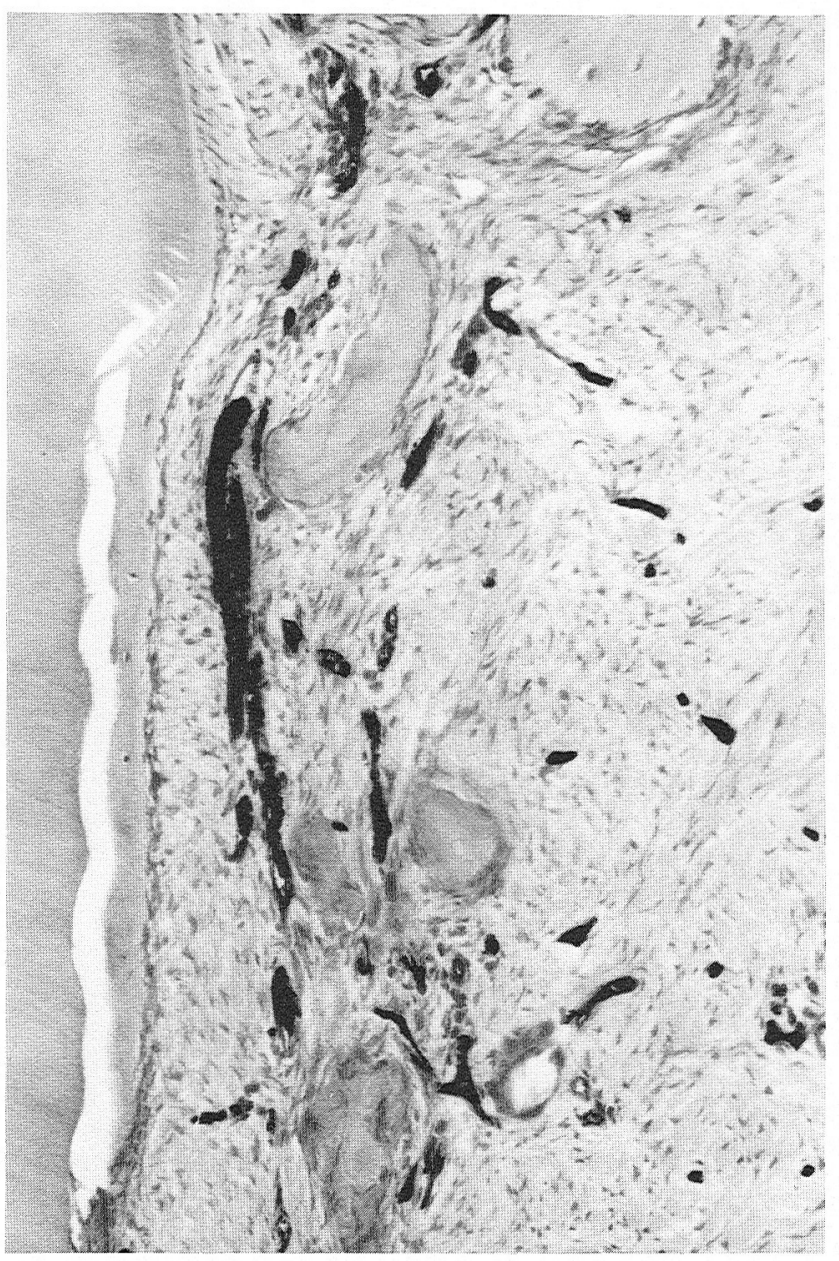

Figure 6. Thirty day specimen. Increased vascularization associated with an area of cementum deposition. Hematoxylin and eosin.

periodontal tissues appears normal. The periosteal vascular network, however, continues to be modified by local remodeling processes occurring at the alveolar cortical bone, and alveolar crest (Figs. 7A, 7B).

\section{DISCUSSION}

Good flap adaptation is fundamental to promote a fast, uneventful and uncomplicated healing. ${ }^{1}$ When flaps are properly adapted, a fibrinous clot will help stabilize the tissues in position, and at the same time favor its nutrition.

Where mucoperiosteal flaps are used, the vascularization of the tissues is apparently less traumatized than when a split thickness flap is used. ${ }^{4}$ All these factors contribute to fast healing of the tissues to the tooth and bony surfaces. The findings from the present study fully support this statement. Initially the area of surgical reflection of the tissues from the teeth and bone was readily seen due to the presence of the intervening blood clot. Any vascular connection between the bone and the reflected gingival tissues or between the periodontal membrane and the gingival margin had been severed. However, vascular continuity was evident between the gingival tissues and the cortical bone apical to the level of the reflection of the tissues. Only 1 day after surgery the supraperiosteal network in the flap, as well as the periodontal and bone marrow vessels, were distinctively hyperemic as demonstrated by the full perfusion present.

Vessels differentiating from the cancellous bone and periodontal membrane, as well as from the flap vasculature, started to invade the organizing clot, thus restoring the connections between the involved tissues. In 7 days these connections were already restored. In particular, when thin buccal bone was present, patent vessels were already depicted perfusing through it, connecting the gingival and the periodontal vasculature. As mentioned by Kon et al. ${ }^{3}$ this hyperemic response reached its peak after 1 week and then started to decrease. Undoubtedly, good flap adaptation will favor fast reorganization of the tissues after surgery.

In 2 weeks the proliferation and patency of the vascular network was reduced almost to normal levels. However, there were still some isolated hyperemic areas, which persisted after 1 month. These foci of persistent hyperemia were associated with either areas of bone remodeling, localized foci of marginal inflammation or cementum remodeling.

The extent of bone resorption observed under the flap was very minimal. Evidently, good flap adaptation protected the integrity of the underlying bone, and as a consequence the process of remodeling did not result in any significant loss. ${ }^{1}$ The presence of a supraperiosteal network showing highly engorged blood vessels immediately after surgery, may have accounted for the protection of the bone. A close relationship between the osteogenic capacity of the periosteum and its vascularization has been reported. ${ }^{7}$ It has been postulated also that high oxygen concentration would favor the deposition of bone. However, extremely high oxygen concentrations would favor bone resorption. ${ }^{8,9}$ This is the kind of environment that could be created with the extreme vasodilation initially observed in the periosteal vasculature. Initial osteoclasis would then be followed by osteoblastic activity, corresponding to the diminishing density of the periosteal vasculature. In a previous investigation bone resorption was observed approximately 1 week after flap surgery, while afterwards bone deposition took place. ${ }^{1}$ This time table corresponds closely to the changes observed in the vasculature in the present report. The influence of the vasculature could be related to the areas of cementum remodeling found after 15 days which were always associated with dilated vessels in the adjacent connective tissue.

Persistent areas of inflammation have been described associated with poorly adapted flaps especially when healing took place by third intention. ${ }^{1}$ Extension of the inflammation within the periodontal membrane was accompanied by areas of bone resorption and root resorption. The foci of persistent inflammation found in this study were almost invariably associated with areas where good flap adaptation was not achieved. As expected, these areas depicted engorged vessels, and were associated with zones undergoing delayed remodeling. 


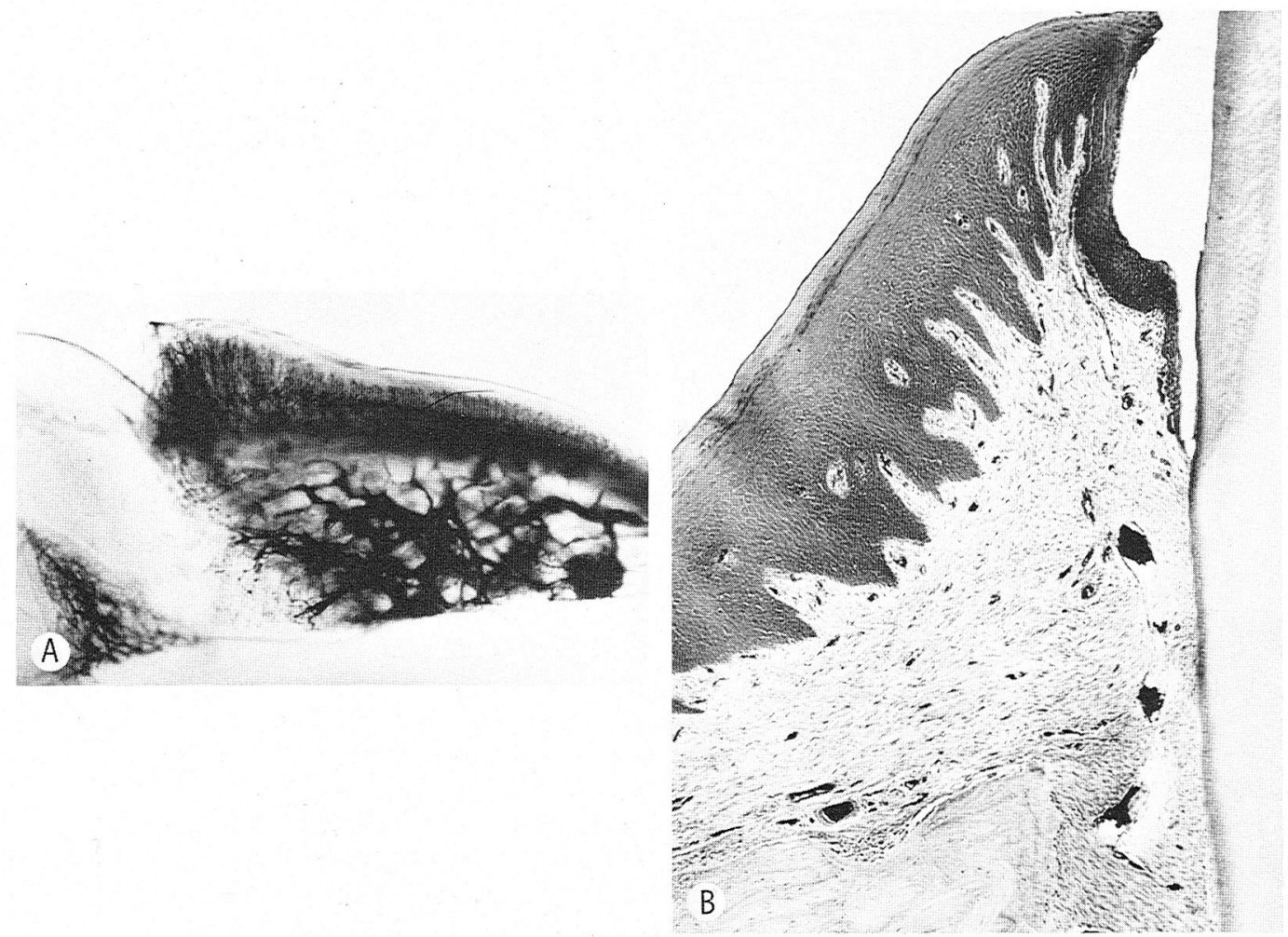

Figure 7. Ninety day specimen. A. Cleared specimen. The vasculature of the periodontal tissues has been restored. B. Histologically, tissues look normal. An area of bone remodeling is still present at the alveolar crest. Hematoxylin and eosin.

Similarly, areas of persistent hyperemia have been described, using the same India ink injection technique, after tooth replantation. ${ }^{10}$ In that instance, the hyperemic foci were associated with postoperative complications such as ankylosis, delayed remodeling or development of periapical processes.

It is evident that the better the flap adaptation obtained, the faster normal vascularization will be reestablished, thus minimizing complications during the healing of the tissues to the teeth and underlying bone.

\section{CONCLUSIONS}

1. Vascular anastomoses will be restored early in the healing period after mucoperiosteal flap surgery.

2. Good flap adaptation will promote rapid revascularization.

3. Isolated hyperemic foci may remain associated with areas of marginal inflammation or delayed remodeling.

\section{REFERENCES}

1. Caffesse, R. G., Ramfjord, S. P., and Nasjleti, C. E.: Reverse bevel periodontal flaps in monkeys. $J$ Periodontol 39: 219, 1968.

2. Lahiffe, B. J., Caffesse, R. G., and Nasjleti, C. E.: Healing of periodontal flaps following use of MBR 4197 (Flucrylate) in Rhesus monkeys. A clinical and histological evaluation. $J$ Periodontol 49: 635, 1978.

3. Kon, S., Novaes, A., Ruben, M., and Goldman, H.: Visualization of the microvascularization of the healing periodontal wound. IV. Mucogingival surgery: Full thickness flap. J Periodontol 40: 441, 1969.

4. Tissot, R., and Sullivan, H.: Evaluation of survival of partial thickness and full thickness flaps. I.A.D.R. 49th General Meeting, Abstr. 470, p 170, 1971.

5. Ramfjord, S. P., and Nissle, R.: Modified Widman flap. $J$ Periodontol 45: 601, 1974.

6. Spalteholtz, W.: Die Arterien der Herzwand, p 13. Leipzig, S. Hirzel, 1924.

7. Cavadies, A., and Trueta, J.: An experimental study of the vascular contribution to the callus of fractures. Surg Gyn Obs 120: 731, 1965.

8. Girgis, F., and Pritchard, J.: Experimental contribution of cartilage during the repair of fractures of skull vaults in rats. $J$ Bone Joint Surg 40B: 274, 1958.

9. Basset, A.: Clinical implications of cell function in bone grafting. Clin Orthop 87: 49, 1972.

10. Castelli, W. A., Nasjleti, C. E., Caffesse, R. G., and Diaz-Perez, R.: Vascular response after replantation of teeth. Oral Surg (In press).

Send reprint requests to: Dr. Raul G. Caffesse, The University of Michigan School of Dentistry, Ann Arbor, MI 48109. 